
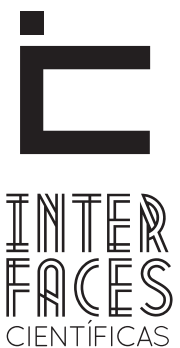

SAÚDE E AMBIENTE

\title{
ANÁLISE DA CAPACIDADE FÍSICA E RESPIRATÓRIA DE ALUNAS PRATICANTES DO MÉTODO PILATES NO MUNICÍPIO DE ARACAJU/SE
}

Adely Amaly de Oliveira Rocha ${ }^{1}$

Aida Carla Santana de Melo Costa ${ }^{3}$

Fabiana Soares Luduvice ${ }^{2}$

\section{RESUMO}

A funcionalidade constitui a habilidade física necessária para a realização de tarefas do cotidiano. 0 presente trabalho teve como objetivo analisar a capacidade funcional de alunas praticantes do método Pilates no município de Aracaju/SE. Este estudo foi de caráter transversal, observacional e de campo, sob abordagem quali-quantitativa, realizado no período de abril a maio de 2012. A avaliação foi realizada na primeira e oitava sessões de Pilates, sendo coletados os seguintes parâmetros: saturação parcial de oxigênio, frequência cardíaca, frequência respiratória, pressão arterial, pico de fluxo expiratório, volume inspiratório máximo e a performance física funcional. Foram observadas alterações estatisticamente significativas no índice de massa corpórea, na frequência respiratória, no volume inspiratório máximo e no questionário de performance física após as sessões de Pilates.

\section{PALAVRAS-CHAVE}

Capacidade Física. Capacidade Respiratória. Alunas. Método Pilates. 


\section{ABSTRACT}

The functionality is the physical ability required to perform daily tasks. This study aimed to analyze the functional capacity of practitioners students of Pilates in the city of Aracaju / SE. This study was a cross-sectional, observational and field, in qualitative and quantitative approach, performed from April to May 2012. The evaluation was performed in the first and eighth Pilates sessions, with the following parameters collected: oxygen saturation, heart rate, respiratory rate, blood pressure, peak expiratory flow,

\section{RESUMEN}

La funcionalidad es la capacidad física para realizar las tareas diarias. Este estudio tuvo como objetivo analizar la capacidad funcional de los estudiantes que practican Pilates en la ciudad de Aracaju. Esta investigación fue transversal, observacional función y el campo, bajo un enfoque cualitativo y cuantitativo, realizado en el período de abril a mayo de 2012. La evaluación se realizó en la primera y octava sesiones de Pilates y se recogieron los siguientes parámetros: la saturación de oxígeno, la frecuencia cardiaca, la frecuencia respiratoria, la presión arterial, el flujo maximal inspiratory volume and functional physical performance. Statistically significant changes were observed in body mass index, respiratory rate, maximum inspiratory volume in the questionnaire of physical performance after the Pilates sessions.

\section{KEYWORDS}

Physical Capacity. Respiratory Capacity. Students. Pilates Method. espiratorio máximo, el volumen inspiratorio máximo y el rendimiento físico funcional. Se observó una diferencia estadísticamente significativa en el índice de masa corporal, la frecuencia respiratoria, el volumen inspiratorio máximo y en la prueba de rendimiento físico después de las sesiones de Pilates.

\section{PALAVRAS CLAVE}

Capacidad Física. La Capacidad Respiratoria. Estudiantes. Método Pilates. 


\section{INTRODUÇ̃̃̃O}

O método Pilates foi originalmente desenvolvido por Joseph Pilates durante a Primeira Guerra Mundial e levado para os EUA em 1923 (MUSCOLINO; CIPRIANI, 2004). A experiência de trabalho no Pilates é caracterizada pelo uso de aparelhos diferenciados em que a sobrecarga externa imposta à estrutura musculoesquelética é obtida pelo auxílio de molas (RYDEARD et al., 2006).

Esse método vem ganhando popularidade nos últimos anos, sendo utilizado para condicionamento físico (SEGAL et al., 2004; JAGO et al., 2006) e programa de reabilitação (BLUM, 2002; KOLYNIAK et al., 2004; LA TOUCHE et al., 2008), uma vez que sugere proporcionar alterações nos músculos envolvidos na respiração, consequentemente, melhora da coordenação respiratória e fortalecimento intenso da musculatura abdominal e dos demais músculos inseridos no tronco, ocorrendo mudanças na função respiratória (PANELLI; DE MARCO, 2009). No Pilates, o diafragma é trabalhado, levando a um relaxamento e gerando uma postura correta (MIGUEL, 2007).

No princípio, é comum respirarmos de forma errada, utilizando apenas uma parte de nossa capacidade pulmonar. Então, o método Pilates visa enfatizar a respiração no início do movimento, fornecendo organização do tronco e favorecendo os músculos inspiratórios e cervicais. Ocorrendo de forma sincronizada com a ação muscular, favorece a ventilação pulmonar e melhora a oxigenação tecidual (PIRES; SÁ, 2005).

Os músculos respiratórios apresentam maior fluxo sanguíneo, densidade capilar aumentada, tornando-se mais resistentes à fadiga do que outros músculos esqueléticos. Então, o treinamento da força dos músculos respiratórios pode melhorar a mecânica ventilatória, com o aumento da complacência e diminuição na resistência das vias aéreas. 0 treinamento de endurance pode ser útil para retardar ou prevenir a fadiga muscular ventilatória (FORGIARINI et al., 2010).
A capacidade física pode ser definida como todo o atributo "treinável" no organismo, ou seja, passível de adaptações, sendo composto por variáveis fisiológicas como potência aeróbica máxima, força, flexibilidade e componentes da composição corporal, assim como agilidade, equilíbrio, coordenação motora e velocidade são mais valorizadas, objetivando o desempenho desportivo. Estudos comprovam que quanto mais ativa uma pessoa, menos limitações físicas ela tem (ARAÚJO, 2000).

0 interesse em desenvolver esta pesquisa surgiu devido à escassez de estudos com base em um número consideravelmente menor de publicações nacionais, além da ausência de estudo dessa natureza no município de Aracaju. Este estudo justifica-se, também, pela necessidade de analisar os efeitos do método Pilates na função motora e respiratória em praticantes desta modalidade.

O objetivo deste estudo foi analisar a capacidade física e respiratória, por meio de marcadores funcionais e do teste de performance física em alunas praticantes do método Pilates.

\section{METODOLOGIA}

Esta pesquisa é de caráter descritivo, prospectivo, observacional e de campo, com natureza quali-quantitativa, realizada no estúdio Top Pilates, localizado no município de Aracaju/SE. O projeto foi submetido ao Comitê de Ética em Pesquisa da Universidade Tiradentes e os termos da Resolução 196/96 do CNS/MS foram respeitados, assim como a regulamentação para a realização de pesquisa clínica, legislada pela ANVISA.

Foram avaliadas 42 mulheres, sendo que uma delas apresentava apneia do sono e era submetida à ventilação não invasiva (CPAP) e outras três não concluíram as sessões propostas para o estudo. Essas sessões correspondiam a um mês de atividade, o equivalente a oito aulas, 
sendo realizadas duas vezes por semana, com duração de 50 minutos, ministradas sempre pelo mesmo instrutor. Dessa forma, a amostra foi constituída por 38 alunas.

Utilizou-se como critérios de inclusão praticantes do método Pilates por mais de seis meses ininterruptos, do gênero feminino, com faixa etária adulta, não praticantes de outra atividade física, admitidas no estúdio Top Pilates no município de Aracaju/SE. Como fatores de exclusão, foram considerados indivíduos sedentários, do gênero masculino, cardiopatas e/ou pneumopatas. A amostra foi estratificada em três grupos, sendo o primeiro composto por alunas com idade menor ou igual a 30 anos; o segundo, com faixa etária entre 31 e 59 anos; e o terceiro grupo representado por mulheres com idade igual ou maior que 60 anos, considerando-se as variações fisiológicas em cada faixa etária estudada.

Os dados foram coletados, inicialmente, por meio de uma ficha de avaliação previamente elaborada pelas próprias pesquisadoras, contendo dados pessoais, como nome completo da voluntária, gênero, idade, estado civil, profissão, raça, peso, altura e índice de massa corpórea (IMC).

Foi feita uma submissão à coleta de dados antes do início da atividade Pilates acerca da capacidade física por meio do Teste de Performance Física, pico de fluxo expiratório (Peak Flow ${ }^{\circledR}$ ), volume inspiratório máximo (Voldyne $\left.{ }^{\circledR}\right)$, frequências cardíaca e respiratória, pressão arterial e saturação parcial de oxigênio. A avaliação foi feita em dois momentos: antes e depois das oito sessões de Pilates, com frequência de duas aulas semanais.

Para dar início à coleta de dados, as alunas estavam em um ambiente reservado, calmo, realizando a avaliação do pico de fluxo expiratório, utilizando o Peak Flow Meter Assess - Reorder No 710 Full Range (60-880 l/min) e do volume inspiratório máximo com o Voldyne $5000^{\circledR}$ da marca Hudson $\mathrm{RCl}$, EUA.

As alunas estavam em repouso, sentadas em uma cadeira com os pés apoiados, membros superiores em cadeia fechada para evitar utilização da musculatura acessória, a cabeça em posição neutra, sendo solicitado que as mesmas realizassem uma inspiração máxima até a capacidade pulmonar total e, em seguida, um sopro rápido e mais forte possível para avaliação do pico de fluxo expiratório. Para verificar o volume inspiratório máximo, elas foram orientadas a realizar uma expiração forçada seguida de uma inspiração máxima sustentada, utilizando um clipe nasal de modo que não houvesse escape de ar, sendo essa medida realizada três vezes com cada paciente e considerando o maior valor obtido.

A mensuração da pressão arterial foi iniciada com o paciente sentado, braço esquerdo apoiado, utilizando esfingmomanômetro com manguito específico para adultos de marca aneróide Premium e com uso de estetoscópio BD duo-sonic.

Para medir a saturação parcial de oxigênio e a frequência cardíaca, foi utilizado um oxímetro de pulso digital da marca UMP, onde a paciente permanecia sentada em uma cadeira, de maneira confortável e sem a utilização de esmaltes na unha para que não houvesse interferência na mensuração.

A frequência respiratória foi observada pelas pesquisadoras de modo visual, contando a respiração durante 60 segundos, com a paciente sentada de maneira confortável, sem que a mesma tivesse conhecimento do procedimento que estava sendo realizado, a fim de não interferir no valor desse sinal vital.

O teste de performance física funcional de dez tarefas (PFP-10) consiste em um teste de campo de medidas válidas e confiáveis, usado para quantificar a capacidade funcional avaliada por meio de dez tarefas cotidianas. Estas são desenvolvidas no esforço máximo do indivíduo dentro do seu julgamento de conforto e segurança. É classificada em três níveis: baixa, moderada e alta dificuldade, realizadas sequencialmente, sem interrupção, sendo o tempo total da realização das tarefas um desfecho de endurance (STANZANI, 2009). 
O nível baixo desse teste consiste em conduzir o peso em uma panela entre um balcão e outro (1m), colocar e retirar um casaco, colocar e remover um objeto de uma estante; o nível moderado corresponde a varrer uma porção de areia com uma vassoura e transferir para uma pá, transferir roupas de uma lavadora para secadora e da secadora para uma cesta, pegar quatro lenços do chão; já o nível alto visa conduzir compras em sacolas por 52,3 metros, sentar-se e levantar-se do chão e subir e descer escadas de oito degraus (STANZANI, 2009).

Esse teste foi realizado em forma de perguntas específicas destinadas às alunas, a fim de sabermos se as mesmas conseguiam executar as tarefas, pois elas apresentam uma função cognitiva preservada. Com relação ao último tópico, teste de caminhada de seis minutos, optou-se por não avaliá-lo em decorrência da pouca aceitação das alunas quanto à sua realização, alegando que demandaria tempo e comprometeria a aula que estavam prestes a realizar.

A tabulação de dados e tabelas foi confeccionada pelo Programa Microsoft Excel 2010. Os gráficos e estatística foram realizados por meio do Programa GraphPad Prim 5.0. O teste de Kolmogorov-Smirnov foi utilizado para analisar a normalidade das variáveis estudadas. Para os dados que seguiram distribuição normal, utilizou-se o teste $t$-Student pareado e independente. Para os grupos em que a suposição de normalidade não foi satisfeita, foram aplicados os testes Wilcoxon Marched Pairs e Mann-Whitney para amostras pareadas e independentes, respectivamente. $\mathrm{Na}$ análise das médias de mais de duas amostras não relacionadas entre grupos, utilizou-se o teste ANOVA ONE WAY e o pós-teste de Tukey. Para fazer a correlação entre as amostras estudadas, utilizou-se o teste de Pearson. Em todas as análises, foi utilizado o nível de significância de $95 \%$.

\section{RESULTADOS}

Nesta pesquisa, das 38 alunas submetidas às oito sessões de Pilates, 5 (13,16\%) apresentavam faixa etária menor que 30 anos, 23 (60,53\%) entre 31 e 59 anos e 10 (26,31\%) acima de 60 anos. Em relação ao peso corporal, observou-se diferença estatisticamente significativa na faixa etária 31-59 anos $(p<0,05)$. Quanto ao IMC, foi evidenciada significância estatística nas faixas etárias de $31-59$ anos $(p<0,01)$ e acima de 60 anos $(p<0,001)$ (Tabela 1).

Tabela 1 - Dados demográficos de idade, peso e índice de massa corpórea (IMC) de mulheres adultas distribuídos nos três grupos de faixa etária submetidas a oito sessões de Pilates

\begin{tabular}{cccccccc}
\hline 30 anos & $\begin{array}{c}\text { Média e } \\
\text { desvio-padrão }\end{array}$ & $\begin{array}{c}31 \text { à } 59 \\
\text { anos }\end{array}$ & $\begin{array}{c}\text { Média e } \\
\text { desvio-padrão }\end{array}$ & Valor de $\mathrm{p}$ & $\geq 60$ anos & $\begin{array}{c}\text { Média e } \\
\text { desvio-padrã }\end{array}$ & Valor de $p$ \\
\hline Idade & $\begin{array}{c}26,80 \pm 0,8 \\
3\end{array}$ & Idade & $47,15 \pm 8,64$ & - & Idade & $65,30 \pm 3,77$ & - \\
Peso & $\begin{array}{c}53,60 \pm 4,2 \\
1^{\text {a }}\end{array}$ & Peso & $65,59 \pm 9,6^{\mathrm{a}}$ & $0,05^{*}$ & Peso & $62,85 \pm 9,31$ & - \\
IMC & $\begin{array}{c}21,00 \pm 1,8 \\
7^{b, c}\end{array}$ & IMC & $25,1 \pm 2,93^{b}$ & $0,01^{* *}$ & IMC & $26,50 \pm 3,34^{c}$ & $0,001^{* * *}$ \\
\hline
\end{tabular}

Fonte: Dados da pesquisa, 2014.

${ }^{*} p<0,05,{ }^{* *} p<0,01, * * * p<0,001$ em valor do teste ANOVA ONE WAY e pós-teste Tukey para comparação de médias em amostras não pareadas. 
Na Tabela 2, observou-se que não houve alteração estatisticamente significativa relacionada à pressão arterial entre a primeira e a oitava sessão, bem como em relação às três faixas etárias estudadas.

Tabela 2 - Leitura das médias e desvio-padrão da pressão arterial em mulheres adultas, distribuídos nos três grupos de faixa etária, submetidas a oito sessões de Pilates

$\begin{array}{cc}\text { Grupos } & \begin{array}{c}\text { Tempo de } \\ \text { leitura }\end{array} \\ \begin{array}{c}\text { Média da Pressão Arterial } \\ \text { (Sistólica/Diastólica) }\end{array}\end{array}$

Média e desvio-padrão

\begin{tabular}{llc}
\hline \multirow{2}{*}{ 30 anos } & antes & $95 \pm 6,1$ \\
& depois & $87,5 \pm 6,4$ \\
de 31 a 59 & antes & $99,87 \pm 11,6$ \\
anos & depois & $96,67 \pm 7,1$ \\
& & $102,3 \pm 10,7$ \\
$\geq 60$ anos & antes & $101,7 \pm 9,6$
\end{tabular}

Fonte: Dados da pesquisa, 2014

Média das pressões sistólica e diastólica. Valor de $\mathrm{p}=0,08$ para faixa etária $\leq 30$ anos; $p=0,32$ para 31 a 59 anos e $p=0,7237$ para $\geq 60$ anos.

A Figura 1 demonstra alteração estatisticamente significativa da frequência respiratória na faixa etária menor que 30 anos, quando comparados os dois momentos da avaliação (antes 21,60 e depois 18,80). No entanto, nos demais grupos, não houve diferença estatisticamente significativa.
Figura 1 - Comportamento da frequência respiratória em mulheres adultas, distribuído nos três grupos de faixa etária, submetidas a oito sessões de Pilates

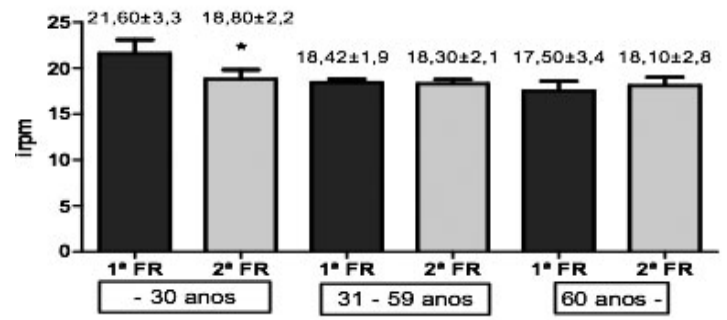

Fonte: Dados da pesquisa, 2014

* $\mathrm{p}=0,0312$ em valor do teste t-pareado para comparação de médias em amostras pareadas, antes e depois de tratados.

Na Figura 2, observou-se que não houve alteração estatisticamente significativa relacionada à freqüência cardíaca entre a primeira e oitava sessão, bem como em relação às três faixas etárias estudadas.

Figura 2 - Comportamento da frequência cardíaca nos três grupos de faixa etária, submetidas a oito sessões de Pilates

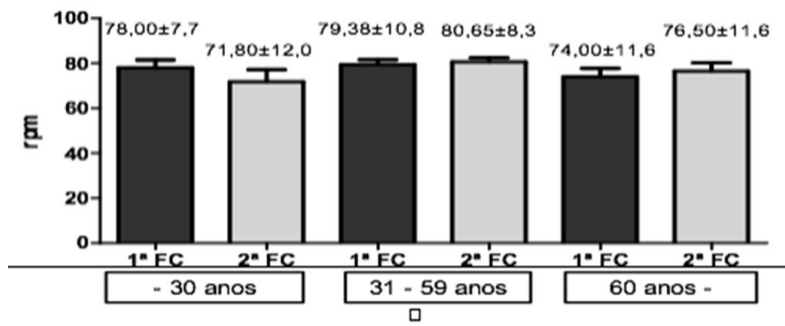

Fonte: Dados da pesquisa, 2014. 
Na Tabela 3, não houve significância estatística relacionada à SpO2 entre a primeira e oitava sessão, bem como em relação às três faixas etárias.

Tabela 3 - Médias e desvio-padrão da SpO2 em mulheres adultas, distribuída nas três faixas etárias, submetidas a oito sessões de Pilates

\begin{tabular}{lccl}
\hline Grupos & $\begin{array}{c}\text { Tempo de } \\
\text { leitura }\end{array}$ & $\begin{array}{c}\text { Média e } \\
\text { desvio-padrão }\end{array}$ & $\begin{array}{l}\text { Valor } \\
\text { de } p\end{array}$ \\
\hline s30 anos & Antes & $98,00 \pm 0,7071$ & - \\
& Depois & $98,80 \pm 0,4472$ & \\
\multirow{2}{*}{31 à 59} & Antes & $98,31 \pm 0,8376$ & - \\
anos & Depois & $98,52 \pm 0,8980$ & \\
& & & \\
$\geq 60$ anos & Antes & $97,60 \pm 2,503$ & - \\
em diante & Depois & $98,70 \pm 0,6749$ &
\end{tabular}

Fonte: Dados da pesquisa, 2014

Teste Wilcoxon para comparação de médias em amostras pareadas, antes e depois de tratados.

Na Figura 3, observou-se que não houve alteração estatisticamente significativa relacionada ao pico de fluxo expiratório máximo entre a primeira e oitava sessão. No entanto, nota-se um fluxo inferior no grupo correspondente à faixa etária acima de 60 anos, em relação aos demais grupos.
Figura 3 - Comportamento do Peak Flow ${ }^{\circledR}$ em mulheres adultas, distribuídos nos três grupos de faixa etária, submetidas a oito sessões de Pilates

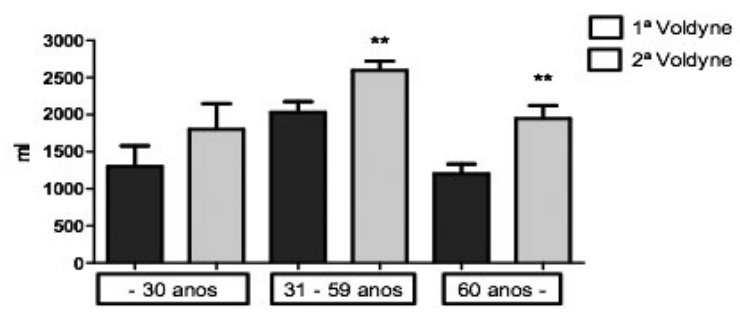

Fonte: Dados da pesquisa, 2014.

Teste Wilcoxon para comparação de médias em amostras pareadas, antes e depois de tratados.

A Figura 4 demonstra diferença estatisticamente significativa do volume inspiratório máximo nas faixas etárias de 31 a 59 anos $(p<0,01)$ e acima de 60 anos $(p<0,01)$. No entanto, no grupo correspondente à faixa etária de menores de 30 anos, não houve diferença significativa.

Figura 4 - Comportamento do Voldyne ${ }^{\circledR}$ em mulheres adultas, distribuídos nos três grupos de faixa etária, submetidas a oito sessões de Pilates

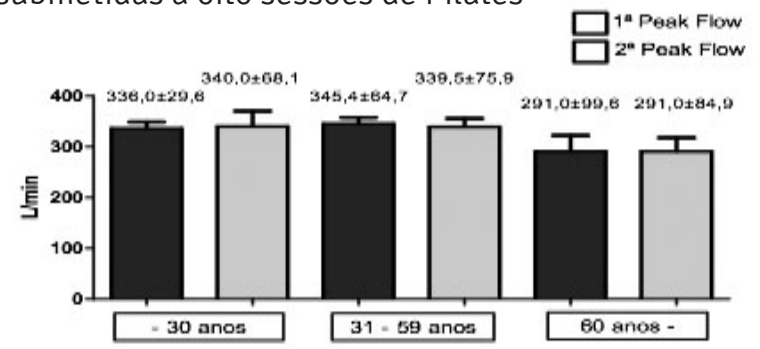

Fonte: Dados da pesquisa, 2014.

** $p<0,01$ em valor do teste de Wilcoxon para comparação em amostras pareadas, antes e depois de tratados. 
Na Tabela 4, observou-se alteração nas respostas do questionário "Tarefas PFP" antes do Pilates nos grupos representados pelas pacientes com mais de 60 anos, $45,45 \%(n=5)$ e pelas pacientes entre $31-59$ anos, 3,84\% ( $n=1)$. Após o Pilates, apenas $27,27 \%(n=3)$ do grupo com mais de 60 anos permaneceram sem realizar a tarefa e nenhuma do grupo de 31-59 anos.

Tabela 4 - Leitura das respostas ao questionário “Tarefas PFP-10" em mulheres adultas nos três grupos de faixa etária, submetidas a oito sessões de Pilates

\begin{tabular}{|c|c|c|c|c|c|}
\hline \multirow[t]{2}{*}{ Intensidade de dificuldade/perguntas } & \multicolumn{2}{|c|}{$1^{a}$ Avaliaçã } & \multicolumn{2}{|c|}{$2^{\mathrm{a}}$ Avaliação } & \multirow[t]{2}{*}{ Valor p } \\
\hline & SIM & NÂO & SIM & NÂO & \\
\hline \multicolumn{6}{|l|}{ Baixa dificuldade } \\
\hline $\begin{array}{l}\text { Conduzir peso numa panela entre um balcão } \\
\text { e outro }(1 \mathrm{~m})\end{array}$ & 36 & 1 & 36 & 1 & \\
\hline Colocar e retirar um casaco & 36 & 1 & 37 & 0 & $(0,0001) * * *$ \\
\hline Colocar e remover um objeto de uma estante & 37 & 0 & 37 & 0 & \\
\hline \multicolumn{6}{|l|}{ Moderada dificuldade } \\
\hline $\begin{array}{l}\text { Varrer } 1 / 2 x . \text { de areia com uma vassoura e } \\
\text { transferir para uma pá }\end{array}$ & 37 & 0 & 37 & 0 & \\
\hline $\begin{array}{l}\text { Transferir roupas de uma lavadora para seca- } \\
\text { dora; e da secadora para uma cesta }\end{array}$ & 37 & 0 & 37 & 0 & \\
\hline Pegar quatro lenços do chão & 37 & 0 & 37 & 0 & \\
\hline \multicolumn{6}{|l|}{ Elevada dificuldade } \\
\hline $\begin{array}{l}\text { Conduzir compras em sacolas por } 52,3 \text { me- } \\
\text { tros }\end{array}$ & 34 & 3 & 35 & 2 & $\begin{array}{l}(0,012)^{*} \\
(0,003)^{* *}\end{array}$ \\
\hline Sentar-se e levantar-se do chão & 34 & 3 & 34 & 3 & $\begin{array}{l}(0,0001) * * * \\
(0,0121)^{*} \\
(0,003)^{* *}\end{array}$ \\
\hline Subir e descer escadas (8 degraus) & 37 & 0 & 37 & 0 & \\
\hline
\end{tabular}

Fonte: Dados da pesquisa, 2014.

*p0,05; **p0,01; ***p0,001. Teste Correlação de “Pearson r" para comparação em amostras pareadas, antes e depois das oito sessões de Pilates. 


\section{DISCUSSÃO}

Segundo Casalini (2011), os homens sentem prazer em realizar atividades físicas, mas não necessariamente em ambientes fechados, ou dividindo o espaço com as mulheres. As mulheres, ao contrário, sentem-se motivadas, planejam sua participação e aguardam os encontros para as atividades com muita expectativa, provavelmente, a maior participação das mulheres em grupos de convivência tem a ver com a possibilidade de uma maior vivência social em espaços que dispõem de ambientes fechados, mais controlados e seguros. Nesta pesquisa, optou-se por avaliar pessoas do gênero feminino em virtude de sua maior demanda no estúdio de Pilates onde foi realizada a pesquisa.

De acordo com Reis e outros autores (2011), no Brasil, há uma maior quantidade de mulheres na faixa etária idosa e esta diferença acentua-se de acordo com o avanço da idade. Essa superioridade em números de mulheres idosas praticantes de Pilates pode ser atribuída à maior tendência ao autocuidado. Porém, o método Pilates ajuda a manter um peso corporal normal, ou se combinado com redução moderada de ingesta calórica, permite redução de peso (MIRANDA; MORAIS, 2009). Por isso, atualmente, há grande demanda de jovens de 18 a 30 anos, objetivando ganhar força e hipertrofia muscular em academias de musculação e não em estúdios de Pilates (AMORIM, 2010).

Matsudo e outros autores (2002) relatam que, com o avanço da idade, há uma prevalência de inatividade física. Homens entre 18 e 29 anos tiveram níveis de inatividade física significativamente mais baixos do que homens mais velhos, enquanto mulheres de 75 anos ou mais velhas tiveram significativamente níveis mais altos de inatividade física do que mulheres mais novas. Isso discorda com o nosso estudo a prevalência de mulheres na faixa etária de 31 a 59 anos e uma redução de procura nas faixas etárias de 18 a 30 anos e de maiores de 60 anos.
Para Rech e outros autores (2006), em pesquisas feitas no Brasil, há aumento significativo na prevalência de excesso de peso e obesidade na população nacional, sendo a prevalência em mulheres. Essa avaliação tem sido comumente realizada por meio de medidas antropométricas de massa corporal e estatura, ou da combinação dessas duas medidas, por apresentar como fatores positivos a praticidade, baixo custo e fácil interpretação dos resultados.

De acordo com Ray e outros autores (1983), nos estados de obesidade (IMC> $30 \mathrm{~kg} / \mathrm{m} 2$ ) sem pneumopatias associadas, encontra-se redução da complacência por deposição do tecido adiposo subcutâneo, desvantagem mecânica dos músculos respiratórios e aumento do volume sanguíneo pulmonar. Segundo Barreto (2002), em um grupo de pacientes com obesidade mórbida, foi encontrado aumento do volume residual e redução dos fluxos máximos a baixos volumes pulmonares, sugerindo obstrução de vias aéreas periféricas com aprisionamento aéreo. Nesta pesquisa, o excesso de peso, observado através do IMC entre 31 e 59 anos $(25,1 \pm 2,93)$ e em maiores que 60 anos $(26,50 \pm 3,34)$ nas alunas praticantes de Pilates poderia justificar uma redução na capacidade respiratória nessas faixas etárias.

Estudos como o de Lopes e outros autores (2005) comprovam que há um aumento do volume minuto durante todo o exercício e um dos fatores é por meio da frequência respiratória, portanto em exercícios de baixa intensidade há aumento da frequência respiratória. Em contrapartida, observou-se na atual pesquisa uma média e desvio padrão da frequência respiratória em menores de 30 anos (antes 21,60 irpm e depois 18,80 irpm), enquanto que nas outras faixas etárias com idade entre 31 e 59 anos (antes 18,42 irpm e depois 18,30 irpm) e com faixa etária maior que 60 anos (antes 17,50 irpm e depois 18,10 irpm), ela se manteve.

Para Carrara e outros autores (2009), os valores normais da SpO2 em ar ambiente estão entre 95 e 
$100 \%$. Em nossa pesquisa, foram encontrados valores mantidos de oximetria em todas as faixas etárias avaliadas, uma vez que as alunas já apresentavam níveis ótimos de saturação parcial de oxigênio desde o momento inicial do estudo.

Segundo Monteiro e Sobral (2004), o exercício físico realizado regularmente promove importantes adaptações autonômicas e hemodinâmicas que vão influenciar o sistema cardiovascular. Há aumento no débito cardíaco, redistribuição no fluxo sanguíneo e elevação da perfusão circulatória para os músculos em atividade. Com isso, a pressão arterial sistólica (PAS) aumenta diretamente na proporção do aumento do débito cardíaco. A pressão arterial diastólica (PAD) reflete a eficiência do mecanismo vasodilatador local dos músculos em atividade. A resistência total ao fluxo sanguíneo cai drasticamente quando o exercício começa, alcançando um mínimo ao redor de $75 \%$ do VO2 máximo. Os níveis tensionais elevam-se durante o exercício físico e no esforço predominantemente estático.

Em 44 estudos randomizados e controlados ( $n=2.674)$, Fagard (2001) avaliou a relação exercício e pressão arterial em repouso, encontrando redução da média da pressão sistólica $(3,4 \mathrm{mmHg}$ em geral; 2,6 $\mathrm{mmHg}$ em normotensos; 7,4 $\mathrm{mmHg}$ em hipertensos) e da pressão diastólica (2,4 mmHg em geral; 1,8 $\mathrm{mmHg}$ em normotensos; 5,8 mmHg em hipertensos). Kokkinos e Papademetriou (2000) relataram reduções de $10 \mathrm{mmHg}$ na pressão sistólica e de $7,5 \mathrm{mmHg}$ na pressão diastólica com treinamento. Em contrapartida, no presente estudo, não houve alteração estatística em relação à pressão arterial, uma vez que as alunas não apresentavam nenhum tipo de cardiopatia associada, mantendo seus níveis pressóricos apropriados mesmo antes de iniciarem a atividade proposta pela pesquisa.

Já Porto (2004) afirma que a pressão arterial varia muito e depende de diferentes fatores que podem alterar seus valores, como idade, sexo, raça, sono, emoções, exercício muscular e alimentação. Neste estudo, por ser mensurada antes da realização dos exercícios, não houve alteração considerável entre a primeira e a última sessão e também em virtude do tempo reduzido de treinamento físico.

Segundo Teixeira e Perreira (2008) a função pulmonar tende a aumentar durante a adolescência, estabilizar-se por volta dos 30 anos e declinar gradualmente depois disso. Esse declínio segue um padrão relacionado à idade, porém, quando há redução entre 40 e 50 anos de idade, pode estar associado a alguns fatores, como o aumento no peso corporal, ao invés de estar ligada a alterações reais nos tecidos.

De acordo com Barreto (2002) a performance funcional pulmonar máxima é conseguida nas idades de 20 anos na mulher e 25 anos no homem, aproximadamente. Após, começa uma lenta e progressiva redução da capacidade funcional pulmonar, que se mantém, entretanto, em condições de proporcionar um adequado intercâmbio de gases, mesmo em idades extremas em indivíduos saudáveis. Esse fato coincide com esta pesquisa, visto que foram observados valores mais satisfatórios em mulheres com faixa etária inferior a 30 anos, em relação aos marcadores funcionais mensurados.

Segundo Conil e outros autores (2003), comparando o valor obtido do pré-teste e os valores obtidos do pós-teste, observou-se que a natação promoveu uma melhora na capacidade aeróbica de todas as crianças, demonstrado no resultado PFE após o exercício. Este melhoramento da capacidade respiratória também pode ser encontrado em muitas pesquisas realizadas enfatizando o efeito crônico do exercício. No entanto, em pesquisas que ressaltam o efeito agudo do exercício, esse evento ainda não foi detectado, principalmente com o parâmetro espirométrico do PFE, em consonância com o atual estudo, não sendo encontradas diferenças substanciais para as faixas etárias menor de 30 anos (antes 336,0 e depois 340,0); entre 31 e 59 anos (antes 345,4 e depois 339,5) e em maiores de 60 anos (antes 291,0 e depois 291,0). 
Para Barreto (2002), a capacidade pulmonar total aos 60 anos de idade é $90 \%$ do seu valor aos 20 anos. Apesar de reduzidos em termos absolutos, os percentuais previstos e os limites da normalidade de idosos saudáveis serão adequados, isto é, interpretados como normais devido ao processo fisiológico.

No entanto, esses valores variam de acordo com a realização de atividade física. Tomich e outros autores (2007), ao compararem três tipos de exercícios respiratórios em indivíduos saudáveis, observaram mudanças no volume e tempo do padrão respiratório quando comparados aos iniciais, sendo que a utilização do Voldyne ${ }^{\circledR}$ apresentou melhores resultados, pois permite inspirações lentas e profundas, o que corrobora os nossos resultados que apresentaram significância para a faixa etária de 31 a 59 anos (antes $2031 \mathrm{ml}$ e depois $2596 \mathrm{ml}$ ) e de maiores de 60 anos (antes $1200 \mathrm{ml}$ e depois $1945 \mathrm{ml})$.

De acordo com Andreotti e Okuma (1999), grande porcentagem de pessoas acima de 60 anos tem algum tipo de dificuldade de realizar tarefas cotidianas. Nota-se uma forte tendência à diminuição da atuação do indivíduo no meio em que vive. Isso vem reforçar Rabelo (2002) e Vasconcelos (2004) ao afirmarem que um programa de atividade física pode ser efetivo para a capacidade funcional de pessoas, podendo contribuir certamente para a autonomia do idoso e, sobretudo, para a qualidade de vida. $\mathrm{Na}$ atual pesquisa, as alunas praticantes do método Pilates apresentaram resultados satisfatórios desde o primeiro momento da avaliação em algumas atividades propostas pelo teste de performance física.

\section{CONSIDERACÕES FINAIS}

Diante deste estudo, pode-se observar que houve alterações positivas em relação aos valores obtidos com o índice de massa corpórea, frequência respiratória, volume inspiratório máximo e o teste de performance física. No entanto, não foram observadas mudanças substanciais na mensuração dos parâmetros saturação parcial de oxigênio, pressão arterial e frequência cardíaca após a prática de Pilates.

Isso pode ser justificado pelo pouco tempo de atividade, além do fato de que as alunas já apresentavam esses parâmetros em níveis ótimos, mesmo antes de iniciarem a atividade em relação à primeira e à segunda coleta de dados, sendo necessário, talvez, um tempo mais prolongado dessa atividade para que fosse notada uma melhor resposta nesses marcadores funcionais.

Programas de exercícios por meio do método Pilates colaboram para a melhoria e manutenção da aptidão física de mulheres. A prática regular dessa modalidade de exercícios por mulheres idosas influencia suas vidas de forma positiva, contribuindo para uma maior autonomia e independência, conforme constatado nos resultados desta pesquisa. Entretanto, há necessidade de um número maior de estudos, bem como de um tempo mais prolongado de execução desta atividade, a fim de avaliar os efeitos aqui abordados.

\section{REFERÊNCIAS}

AMORIM, D. Motivação à prática de musculação por adulto jovem do sexo masculino na faixa etária de 18 a 30 anos. Porto Alegre, 2010.

ANDREOTTI, R.A.; OKUMA, S.S. Validação de uma Bateria de Testes de Atividade de Vida Diária para Idosos Fisicamente Independentes. Revista Paulista de Educação Física, v.13, n.1, 1999, p.1-14.

ARAÚJO D, ARAÚJO C, Aptidão física, saúde e qualidade de vida relacionada à saúde em adultos. Rev Bras Med Esporte, v.6, n.5, 2000, p.143-213.

ATS Statement: Guideline for the six-minute walk test. Am J Respir Crit Care Med, v.166, n.1, 2002, p.111-7.

BACHIOCHI, J. Light-to-frequency conversion (Part 
2). Circuit Cellar. The magazine for computer applications. Janeiro de 2005.

BARRETO, S. S. M. Volumes Pulmonares. J Pneumol. 28 (Supl 3), 2002.

BLUM, C. L. Chiropractic and Pilates therapy for the treatment of adult scoliosis. Journal of Manipulative and Physiological Therapeutics, v.25, n.3, 2002, p.1216.

CAHALIN, L. P. et al. The six minute walk test predicts peak oxygen uptake and survival in patients with advanced heart failure. Chest, v.12, n.110, 1996, p.325332.

CAHALIN, L. et al. The relationship of the 6-min walk test to maximal oxygen consumption in transplant candidates with end stage lung disease. Chest, v.2, n.108, 1995, p.452-459.

CAMARÃO, T. Pilates com bola no Brasil. Rio de Janeiro: Alegre, 2005.

CARRARA, D. et al. Oximetria de pulso arterial. Conselho Regional de Enfermagem. São Paulo, 2009.

CASALINI, E. Perfil dos idosos praticantes de Pilates. Universidade Regional do Noroeste do Estado do Rio Grande do Sul. Departamento de Ciências da Vida, 2011.

CONIL, F. P. et al. Training of aerobic and anaerobic fitness in children with asthma. Journal Pediatrie, v.2, n.142, 2003, p.179-184.

ENRIGHT, P. L. The six minute walk test. Respir Care, v.8, n.48, 2004, p.783-785.

ENRIGHT, P. L.; SHERRILL, D. L. Reference equations for the six minute walk in health adults. Am J Respir Crit Care Med, v.01, n.158, 1998, p.1384-1387.
FAGARD RH. Exercise characteristics and blood pressure responses to dynamic physical training. Med Sci Sports Exerc, v.21, n.33 Suppl 6:S484-S492, 2001

FIORE, J. F. et al. Pressões respiratórias máximas e capacidade vital: comparação entre avaliações através de bocal e de máscara facial, J Bras Pneumol, v.6, n. 30, 2004, p. 515-520.

GALLAGHER, S. P.; KRYZANOWSKA, R. O método Pilates de condicionamento físico. 3.ed. São Paulo: The Pilates Studios do Brasil, 2000.

HAMILTON, D. M.; HAENNEL, R. G. Validity and reliability of the six minute walk test in a cardiac rehabilitation popu. J Cardiopulm Rehabil, v.3, n.20, 2000, p.156-164.

HARADA, N. D.; CHIU, V.; STEWART, A. L. Mobility related function in older adults: assessment with a 6-minute walk test. Arch Phys Med Rehabil., v.01, n.80, p.837-41, 1999.

JAGO, R. et al. Effect of 4 weeks of Pilates on the body composition of young girls. Preventive Medicine, v.42, n.3, 2006, p.177-180.

KOKKINOS, PF; PAPADEMETRIOU, V. Exercise and hypertension. Coron Artery Dis, v.01 n.11, 2000, p.99102 ,

LA TOUCHE, R.; ESCALANTE, K.; LINARES, M. T. Treating non-specific chronic low back pain through the Pilates Method. Journal of Bodywork and Movement Therapies, v.12, n. 103, 2008, p.364-370.

LOPES, R; BRITO, R; PARREIRA, V. Padrão respiratório durante o exercício - revisão literária, R. Brás. Ci e Mov, v.2, n.13, 2005, p.153-160.

MATSUDO, S. Envelhecimento, atividade física e saúde. R. Min. Educ. Fís., Viçosa, v.10, n.1, 2002, p.195-209. 
MATSUDO, S. et al. Physical activity level of São Paulo State population: an analysis based on gender, age, socio-economic status, demographics and knowledge, Rev. Bras. Ciên. e Mov. Brasília, v.10, n.4 p. 41-50, 2002.

MATSUDO, S.M.; MATSUDO, V.K.R.; ARAÚJO, T.L. Perfil do nível de atividade física e capacidade funcional de mulheres maiores de 50 anos de idade de acordo com a idade cronológica. Revista Brasileira de atividade Física e Saúde. v.6, n.1, 2001, p.12-24.

MIGUEL, A. Método Pilates - Benefícios para a terceira idade. Revista Pilates. Abril, 2007.

MIRANDA, L.; MORAES, P. Efeito do método Pilates sobre a composição corporal e flexibilidade, Revista Brasileira de Prescrição e Fisiologia do Exercício, v.3, n.13, 2009, p.16-21.

MONTEIRO, M.; SOBRAL, D. Ejercício físico y el control de la presion arterial, Rev Bras Med Esporte, v.10 n.6, 2004, p.24-35.

PANELLI, C.; DE MARCO, A. Método Pilates de condicionamento do corpo: um programa para toda vida. 2.ed. São Paulo: Phorte, 2009.

PARREIRA, V. F. et al. Pressões respiratórias máximas: valores encontrados e Preditos em indivíduos saudáveis. Rev Bras Fisio, v.11, n.5, 2007, p.361-368.

PORTO, C. C. Exame Clínico: bases para a prática médica. 5.ed. Rio de Janeiro: Guanabara Koogan, 2004.

PRESTO, B.; PRESTO, L. D. N. Fisioterapia respiratória: uma nova visão. Rio de Janeiro: Guanabara Koogan, 2003.
RABELO, H.T. Os Efeitos do Treinamento Contra Resistência no Desempenho nas Atividades da Vida Diária de Mulheres Idosas. 2002. Dissertação (Mestrado em Educação Física) - Universidade Católica de Brasília, Programa de Pós-Graduação Stricto Senso em Educação Física, 2002.

RAY, C. S. et al. Effects of obesity on Respiratory Function. Am Rev Respir Dis, v.13, n.128, 1983, p.501-506.

RECH, C. et al. Indicadores antropométricos de excesso de gordura corporal em mulheres, Rev Bras Med Esporte, v.12, n.3, 2006, p.12-16.

REIS, L.; MASCAREENHAS, C.; LYRA, J. Avaliação da qualidade de vida em idosos praticantes e não praticantes de método, C\&D-Revista Eletrônica da Fainor, Vitória da Conquista, v.4, n.1, 2011, p.38-51.

TEIXEIRA, C.; PERREIRA, E. Alterações morfofisiológicas associadas ao envelhecimento humano, Revista Digital - Buenos Aires - Ano 13 - n.124, 2008.

TOMICH G, et al. Breathing pattern, thoracoabdominal motion and muscular activity during three breathing exercises. Braz J Med Biol Res, v.40, n.10, 2007, p.1409-1417.

VASCONCELOS, C.A. Efeitos da atividade Física Orientada na Auto-Avaliação da capacidade Funcional de Mulheres Idosas. 2004. (Monografia) - Envelhecimento. Faculdade Educação Física, Centro Universitário do Leste de Minas. 
1. Fisioterapeuta da prefeitura municipal de Piaçabuçu (AL) e instrutora de Pilates. E-mail: aaor@hotmail.com

2. Fisioterapeuta graduada pela Universidade Tiradentes.

E-mail: fsl@hotmail.com

3. Fisioterapeuta graduada pela Universidade Tiradentes, pós-graduada em Fisioterapia Neurofuncional pela Universidade Gama Filho (UGF/RJ), mestre em Ciências da Saúde pela Universidade Federal de Sergipe (UFS), doutoranAvaliado em: 14 de Novembro de 2014 Aceito em: 1 de Dezembro de 2014 da em Ciências da Saúde pela Universidade Federal de Sergipe (UFS). E-mail: aida-fisio@hotmail.com 